PROCEEDINGS OF THE

AMERICAN MATHEMATICAL SOCIETY

Volume 129, Number 10, Pages 2997-3008

S 0002-9939(01)06137-8

Article electronically published on April 17, 2001

\title{
A BRASCAMP-LIEB-LUTTINGER-TYPE INEQUALITY AND APPLICATIONS TO SYMMETRIC STABLE PROCESSES
}

\author{
RODRIGO BAÑUELOS, RAFAŁ LATAŁA, AND PEDRO J. MÉNDEZ-HERNÁNDEZ
}

(Communicated by Juha M. Heinonen)

\begin{abstract}
We derive an inequality for multiple integrals from which we conclude various generalized isoperimetric inequalities for Brownian motion and symmetric stable processes in convex domains of fixed inradius. Our multiple integral inequality is a replacement for the classical inequality of H. J. Brascamp, E. H. Lieb and J. M. Luttinger, where instead of fixing the volume of the domain one fixes its inradius.
\end{abstract}

\section{INTRODUCTION}

There is a large class of analytic and probabilistic quantities which are domain dependent and which are bounded above or below by the corresponding quantities when the domain is a ball. Among these are integrals of heat kernels and Green's functions, eigenvalues of the Dirichlet Laplacian and moments of exit times of Brownian motion. These inequalities are often called generalized isoperimetric inequalities. (See [15]-[17].) We now describe some concrete examples related to the topic of this paper. First, let $D \subset \mathbf{R}^{n}$ be a domain and let $D^{*}$ be the ball in $\mathbf{R}^{n}$ with the same volume as $D$ and centered at the origin 0 . Let $P_{t}^{D}(z, w)$ and $P_{t}^{D^{*}}(z, w)$ denote the heat kernels of the Dirichlet Laplacian in $D$ and $D^{*}$, respectively. It is well known (see [1] and [15-17]) that

$$
\int_{D} P_{t}^{D}(z, w) d w \leq \int_{D^{*}} P_{t}^{D^{*}}(0, w) d w
$$

for all $z \in D$ and all $t>0$. This inequality is the same as the probabilistic inequality

$$
P_{z}\left\{\tau_{D}>2 t\right\} \leq P_{0}\left\{\tau_{D^{*}}>2 t\right\}
$$

for all $z \in D$ and all $t>0$, where $\tau_{D}$ is the first exit time of the Brownian motion from the domain $D$ and $P_{z}$ is the associated probability measure when this motion starts at $z$. If we denote the first eigenvalue of the Dirichlet Laplacian in $D$ by $\lambda_{D}$,

Received by the editors February 29, 2000.

1991 Mathematics Subject Classification. Primary 30C45.

Key words and phrases. Symmetric stable processes, generalized isoperimetric inequalities, inradius.

The first author was supported in part by NSF grant \# 9700585-DMS.

The second author was supported in part by KBN grant \# 2 PO3 04315.

The third author was supported in part by Purdue Research Foundation grant \# 690-13953149 .

(C)2001 American Mathematical Society 
it is well known that

$$
-\frac{\lambda_{D}}{2}=\lim _{t \rightarrow \infty} \frac{1}{t} \log P_{z}\left\{\tau_{D}>t\right\}
$$

with a similar expression for $\lambda_{D^{*}}$. These inequalities imply, among other things, the classical Rayleigh-Faber-Krahn inequality

$$
\lambda_{D^{*}} \leq \lambda_{D}
$$

Also, it follows by integrating (1) in time that

$$
\sup _{z \in D} \int_{D} G_{D}(z, w) d w \leq \int_{D^{*}} G_{D^{*}}(0, w) d w
$$

where $G_{D}(z, w)$ and $G_{D^{*}}(0, w)$ are the Green functions of $D$ and $D^{*}$, respectively. Inequality (4) is equivalent to $E_{z} \tau_{D} \leq E_{0} \tau_{D^{*}}$ for all $z \in D$, and in fact it follows from (21) that for all nonnegative increasing functions $\varphi$ we have $E_{z} \varphi\left(\tau_{D}\right) \leq E_{0} \varphi\left(\tau_{D^{*}}\right)$.

As sharp as the above inequalities are, they do not have much meaning when the domains have infinite volume. It is known that for a large class of domains what determines if $\sup _{z \in D} E_{z}\left(\tau_{D}\right)$ is finite is the inradius of the domain. For example, let $D$ be any simply connected domain in the plane and let $R_{D}$ be the supremum of the radii of the disks contained in the domain. This quantity is called the inradius of the domain. It is known (see [2]) that there are positive constants $C_{1}, C_{2}, C_{3}$, and $C_{4}$ such that

$$
C_{1} R_{D}^{2} \leq \sup _{z \in D} \int_{D} G_{D}(z, w) d w \leq C_{2} R_{D}^{2}
$$

and

$$
\frac{C_{3}}{R_{D}^{2}} \leq \lambda_{D} \leq \frac{C_{4}}{R_{D}^{2}}
$$

We should note that the lower bound in (5) and the upper bound in (6) are trivial by domain monotonicity. In fact, the corresponding quantities for the disk of radius $R_{D}=1$ give the constants $C_{1}$ and $C_{4}$; the upper bound in (5) and the lower bound in (6) are what is of significance here. There has been considerable interest in obtaining sharp versions of the right-hand side of (5) and the left-hand side of (6) and in identifying the extremal domains. We refer the reader to R. Bañuelos and T. Carroll [2 for some of the extensive literature on this subject and for connections to other problems. These problems seem to be very difficult for arbitrary simply connected domains and conjectures; on how the extremals domains should look is not even available. Even within the class of convex domains these problems are not easy. Here, however, there has been considerable progress.

Let $D \subset \mathbf{R}^{n}$ be a convex domain with $0<R_{D}<\infty$. Set $I(D)=\left(-R_{D}, R_{D}\right)$, the symmetric interval in the real line of inradius $R_{D}$. The infinite slab (infinite strip for $n=2)$ of inradius $R_{D}$ in $\mathbf{R}^{n}$ is the domain $S(D)=\left\{z=\left(x_{1}, \ldots, x_{n}\right)\right.$ : $\left.x_{i} \in \mathbf{R}, 1 \leq i \leq n-1, x_{n} \in I(D)\right\}$. It was proved by J. Hersch [12] $(n=2)$ and by M. Protter [18] $(n \geq 3)$ that

$$
\lambda_{I(D)}=\lambda_{S(D)} \leq \lambda_{D}
$$


R. Sperb [19] proved that

$$
\int_{D} G_{D}(z, w) d w \leq \int_{-R_{D}}^{R_{D}} G_{I(D)}(0, w) d w
$$

for all $z \in D$. Finally, R. Bañuelos and P. Kröger [4] proved that

$$
\int_{D} P_{t}^{D}(z, w) d w \leq \int_{-R_{D}}^{R_{D}} P_{t}^{I(D)}(0, w) d w
$$

for all $z \in D$ and $t>0$. It is important to note here that inequalities (7), (8) and (9) are all false if the convexity assumption of the domain is removed. This can be seen by taking a slit disk. We refer the reader to 2] for details.

Inequality (9) implies, as we have already observed, (7), (8) and also that

$$
E_{z} \varphi\left(\tau_{D}\right) \leq E_{0} \varphi\left(\tau_{I(D)}\right)
$$

for all $z \in D$ and all nonnegative increasing functions $\varphi$. The proof of (9) given in Bañuelos and Kröger 4 is based on the maximum principle technique of L. Payne and on an adaptation of this to the heat equation by Kröger [14. The purpose of this paper is to present a different proof for the case of $D \subset \mathbf{R}^{2}$ based on an inequality for multiple integrals motivated by the now classical result of Brascamp, Lieb and Luttinger [5. Even though our proof at this point only works for domains in $\mathbf{R}^{2}$, it has several advantages over the proof given in [4]. This method not only proves the result for the classical heat equation but the proof applies, without change, to the symmetric stable processes. Indeed, this argument will work for any Levy process that has right continuous paths and whose transition probabilities are radially, symmetric and nonincreasing.

Recall that a function $f$ on $\mathbf{R}^{2}$ is radially symmetric and nonincreasing if $f(z)=$ $\tilde{f}(|z|)$ for some nonincreasing function $\tilde{f}$ on $\mathbf{R}^{+}$. Our isoperimetric-type inequalities will be consequences of the following theorem.

Theorem 1. Let $D$ be a convex domain of finite inradius $R_{D}$ and let $S(D)$ be the infinite strip as defined above. Let $f_{1}, \ldots, f_{m}$ be nonnegative, nonincreasing, radially symmetric functions on $\mathbf{R}^{2}$. Then for any $t_{1}, \ldots, t_{m}>0$ and any $z_{0} \in \mathbf{R}^{2}$ we have

$$
\begin{gathered}
\int_{t_{1} D} \ldots \int_{t_{m} D} \prod_{j=1}^{m} f_{j}\left(z_{j}-z_{j-1}\right) d z_{1} \cdots d z_{m} \\
\leq \int_{t_{1} S(D)} \ldots \int_{t_{m} S(D)} f_{1}\left(z_{1}\right) \prod_{j=2}^{m} f_{j}\left(z_{j}-z_{j-1}\right) d z_{1} \cdots d z_{m} .
\end{gathered}
$$

This Theorem is motivated by the following result which follows from a more general result of Brascamp, Lieb and Luttinger [5].

Theorem 2 ([5]). Let $f_{1}, \ldots, f_{m}$ be nonnegative functions in $\mathbf{R}^{n}$ and let $f_{1}^{*}, \ldots, f_{m}^{*}$ be their symmetric decreasing rearrangements. Then for any $z_{0} \in \mathbf{R}^{n}$ we have

$$
\int_{D^{m}} \prod_{j=1}^{m} f_{j}\left(z_{j}-z_{j-1}\right) d z_{1} \cdots d z_{m} \leq \int_{\left\{D^{*}\right\}^{m}} f_{1}^{*}\left(z_{1}\right) \prod_{j=2}^{m} f_{j}^{*}\left(z_{j}-z_{j-1}\right) d z_{1} \cdots d z_{m} .
$$

As we shall see below, Theorem 2 immediately implies (11), and hence (21), (3)) and (4) not only for Brownian motion but also for any symmetric stable process. In the same way, Theorem 1 will imply (9), and hence also (7) and (8) for Brownian 
motion and for general symmetric stable processes. The proof of Theorem 1 is given in $\S 2$ and the applications are given in $\S 3$. It has been known for several years now that such rearrangement inequalities combined with the probabilistic representation of the heat kernel imply the classical Raleigh-Faber-Krahn inequalities and many other generalized isoperimetric inequalities for heat kernels and Green's functions. See for example [15]-17.

\section{Proof of Theorem 1}

For any convex domain $D \subset \mathbf{R}^{2}$ containing the origin we define the following Minkowski pseudonorm:

$$
\|x\|_{D}=\inf \{t>0: x \in t D\}=\inf \left\{t>0: \frac{1}{t} x \in D\right\} .
$$

To simplify notation we set $S(D)=S$ for the rest of this section. A simple geometric argument shows that any planar convex domain $D$ of finite inradius is contained in a strip of the same inradius or in a triangle $T$ of the same inradius; see [3]. The case of the strip is trivial and hence we may assume that $D$ is an arbitrary triangle $T$. After translating $T$, if necessary, we may assume that its largest incircle is centered at $(0,0)$.

Lemma 1. Let $T$ be a triangle and let $S$ be a strip such that their largest incircles are centered at the origin and have the same inradius. Then for any $z, w \in \mathbf{R}^{2}$ such that $\|z\|_{T}=\|w\|_{S}$ we have

$$
m(T \cap B(z, r)) \leq m(S \cap B(w, r)) \text { for all } r>0,
$$

where $m$ is the Lebesgue measure in $\mathbf{R}^{2}$.

Proof. Let us first set some notation. Let $X, Y, Z$ be three points in $\mathbf{R}^{2}$. We say that a point $W$ in $\mathbf{R}^{2}$ is in the cone $\Gamma(X, Y, Z)$ with vertex at $Y$, if:

- $W$ and $Z$ are in the same closed half plane determined by the line that passes through $X$ and $Y$.

- $W$ and $X$ are in the same closed half plane determined by the line that passes through $Z$ and $Y$.

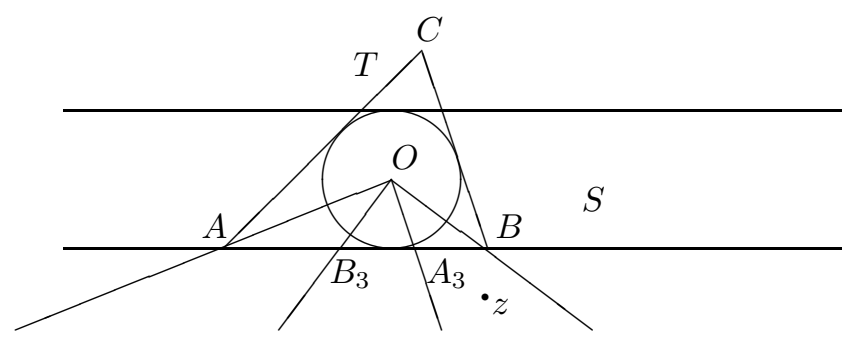

FiguRE 1.

We refer the reader to Figure 1. Without loss of generality we may suppose that $R_{T}=1$. Let $A, B, C$ be the vertices of $T$, and let $z$ be in the cone $\Gamma(A, O, B)$, where 


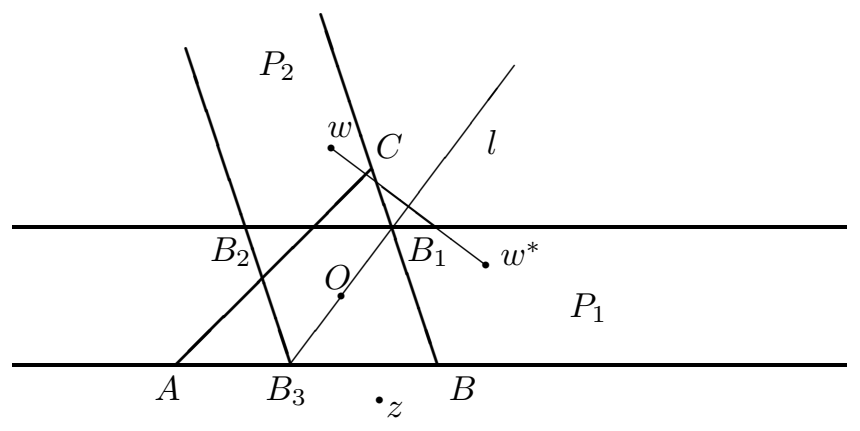

FIGURE 2 .

we have used $O$ to denote the point $(0,0)$. Choose coordinate axes such that the segment $A B$ is situated along $\left\{(x, y) \in \mathbf{R}^{2}: y=-1\right\}$, and $C$ is in the half plane $\left\{(x, y) \in \mathbf{R}^{2}: y>1\right\}$. Then $S$ is the strip $\mathbf{R} \times(-1,1)$ and for $z=\left(x_{1}, y_{1}\right) \neq 0$,

$$
\begin{aligned}
\|z\|_{T} & =\inf \left\{t>0: \frac{1}{t} z \in A B\right\} \\
& =\inf \left\{t>0: \frac{1}{t}\left(x_{1}, y_{1}\right) \in \mathbf{R} \times\{-1\}\right\} \\
& =\left|y_{1}\right|=\|z\|_{S} .
\end{aligned}
$$

Besides, $\|0\|_{T}=\|0\|_{S}=0$. Thus we must prove that

$$
m(T \cap B(z, r)) \leq m(S \cap B(z, r)) \text { for all } r>0 .
$$

Let $B_{1}$ and $A_{1}$ be the points at which the segments $B C$ and $A C$ intersect the line $\left\{(x, y) \in \mathbf{R}^{2}: y=1\right\}$ (see Figure 2). Take $B_{2}$ and $B_{3}$ such that the quadrilateral $B B_{1} B_{2} B_{3}$ is a rhombus with $B(O, 1)$ as its incircle. Note that the segment $B_{2} B_{3}$ is parallel to $B C$ and tangent to $B(O, 1)$. Define $A_{2}, A_{3}$ in a similar way. It is clear that $B_{3}$ and $A_{3}$ are in the segment $A B$, and then the rays $O \vec{B}_{3}$ and $O \vec{A}_{3}$ are in the cone $\Gamma(A, O, B)$.

Now $z$ is either in the cone $\Gamma\left(B_{3}, O, B\right)$ or in the cone $\Gamma\left(A, O, A_{3}\right)$. Suppose $z$ is in $\Gamma\left(B_{3}, O, B\right)$. Let $P_{2}$ be the intersection of the strip given by the segments $B B_{1}$ and $B_{2} B_{3}$ and the half plane $\left\{(x, y) \in \mathbf{R}^{2}: y>1\right\}$, and let $P_{1}$ be the intersection of $S$ with the half plane determined by the line that passes through $B$ and $B_{1}$ which does not contain $O$. Given that $A_{1}$ is in the segment $B_{2} B_{1}$ we have that $T \backslash S \subset P_{2}$.

Thus

$$
m(B(z, r) \cap T \backslash S) \leq m\left(B(z, r) \cap P_{2}\right)
$$

and

$$
m\left(B(z, r) \cap P_{1}\right) \leq m(B(z, r) \cap S \backslash T) .
$$

Hence it is enough to prove that

$$
m\left(B(z, r) \cap P_{2}\right) \leq m\left(B(z, r) \cap P_{1}\right) .
$$

Notice that the reflection of $P_{2}$ with respect to the line $l$ that passes through $B_{1}$ and $B_{3}$ is $P_{1}$. Take $w \in P_{2}$ and let $w^{*}$ be its reflection with respect to $l$. Let $l_{w}$ be the line that passes through $w$ and $w^{*}$. Then $l$ and $l_{w}$ are perpendicular and their intersection is the midpoint of the segment $w w^{*}$. Since $z$ and $w^{*}$ are in the 
same half plane determined by $l$ we have that $|w-z| \geq\left|w^{*}-z\right|$ with equality if and only if $z \in l$. Therefore if $E$ is a Borel set contained in $P_{2} \cap B(z, r)$, then its reflection $E^{*}$, with respect to $l$, is contained in $P_{1} \cap B(z, r)$. By the rotation and translation invariance of the Lebesgue measure we have that $m(E)=m\left(E^{*}\right)$ and thus (10) follows. If $z \in \Gamma\left(A, 0, A_{3}\right)$ we consider the rhombus given by $A, A_{1}, A_{2}, A_{3}$ and follow the same argument. This completes the proof of Lemma 1.

Proof of Theorem 1. First recall that a nonnegative radially symmetric nonincreasing function $f$ can be expressed in the form

$$
f(z)=\int_{0}^{\infty} I_{B(0, r)}(z) d \mu(r)
$$

for some nonnegative measure on $(0, \infty]$. Therefore we may, and will, assume that $f_{i}=I_{B\left(0, r_{i}\right)}$ for some $r_{i}>0$ and $i=1, \ldots, m$. If $m=1$ and $w_{0}$ is such that $\left\|w_{0}\right\|_{S}=\left\|z_{0}\right\|_{T}$ we have by Lemma 1

$$
\begin{aligned}
\int_{t_{1} T} f_{1}\left(z_{1}-z_{0}\right) d z_{1} & =m\left(t_{1} T \cap B\left(z_{0}, r_{1}\right)\right) \\
& \leq m\left(t_{1} S \cap B\left(w_{0}, r_{1}\right)\right) \\
& \leq m\left(t_{1} S \cap B\left(0, r_{1}\right)\right) \\
& =\int_{t_{1} S} f_{1}\left(z_{1}\right) d z_{1} .
\end{aligned}
$$

Now assume that the inequality is true for $m-1$. We have

$$
\begin{gathered}
A:=\int_{t_{1} T} \cdots \int_{t_{m} T} \prod_{j=1}^{m} f_{j}\left(z_{j}-z_{j-1}\right) d z_{1} \cdots d z_{m} \\
=\int_{t_{1} T} \ldots \int_{t_{m-1} T} \prod_{j=1}^{m-1} f_{j}\left(z_{j}-z_{j-1}\right) m\left(t_{m} T \cap B\left(z_{m-1}, r_{m}\right)\right) d z_{1} \cdots d z_{m-1} .
\end{gathered}
$$

Let us define the function $g$ on $\mathbf{R}^{+}$by the formula

$$
g\left(\|z\|_{t_{m} S}\right)=m\left(t_{m} S \cap B\left(z, r_{m}\right)\right) .
$$

Notice that $g$ is well defined, continuous and nonincreasing with $g(\infty)=0$, so

$$
g(t)=\int_{0}^{\infty} I_{[0, s)}(t) d \nu(s)
$$

for some nonnegative measure $\nu$. Hence applying Lemma 1 and the induction assumption

$$
\begin{gathered}
A \leq \int_{t_{1} T} \ldots \int_{t_{m-1} T} \prod_{j=1}^{m-1} f_{j}\left(z_{j}-z_{j-1}\right) g\left(\left\|z_{m-1}\right\|_{t_{m} T}\right) d z_{1} \cdots d z_{m-1} \\
=\int_{0}^{\infty} \int_{t_{1} T} \cdots \int_{t_{m-2} T} \int_{\left(s t_{m} \wedge t_{m-1}\right) T} \prod_{j=1}^{m-1} f_{j}\left(z_{j}-z_{j-1}\right) d z_{1} \cdots d z_{m-1} d \nu(s) \\
\leq \int_{0}^{\infty} \int_{t_{1} S} \cdots \int_{t_{m-2} S} \int_{\left(s \wedge t_{m-1}\right) S} f_{1}\left(z_{1}\right) \prod_{j=2}^{m-1} f_{j}\left(z_{j}-z_{j-1}\right) d z_{1} \cdots d z_{m-1} d \nu(s)
\end{gathered}
$$




$$
=\int_{t_{1} S} \ldots \int_{t_{m} S} f_{1}\left(z_{1}\right) \prod_{j=2}^{m} f_{j}\left(z_{j}-z_{j-1}\right) d z_{1} \cdots d z_{m}
$$

and this proves the theorem.

\section{Applications}

Let $X_{t}$ be an $n$-dimensional symmetric $\alpha$-stable process of order $\alpha \in(0,2]$ and for any Borel set $D \subset \mathbf{R}^{n}$ let $\tau_{D, \alpha}$ be the first exit time of $X_{t}$ from $D$. The process $X_{t}$ has stationary independent increments and its transition density $P_{t}^{\alpha}(z, w)=$ $f_{t}^{\alpha}(z-w)$ is determined by its Fourier transform

$$
\exp \left(-t|z|^{\alpha}\right)=\int_{\mathbf{R}^{n}} e^{i z \cdot w} f_{t}^{\alpha}(w) d w
$$

These processes have right continuous sample paths and their transition densities satisfy the following scaling property:

$$
P_{t}^{\alpha}(z, w)=t^{-\frac{n}{\alpha}} P_{1}^{\alpha}\left(t^{\frac{-1}{\alpha}} z, t^{\frac{-1}{\alpha}} w\right) .
$$

When $\alpha=2$ the process $X_{t}$ is just the $n$-dimensional Brownian motion $B_{t}$ running at twice the speed. That is, if $\alpha=2$, then $X_{t}=B_{2 t}$ and so

$$
P_{t}^{2}(z, w)=P_{2 t}(z, w)=\frac{1}{(4 \pi t)^{n / 2}} \exp \left[\frac{-|z-w|^{2}}{4 t}\right] .
$$

Notice that $\tau_{D, 2}$ is not quite the same as the $\tau_{D}$ from the introduction because of the factor of 2 in the time of the Brownian motion. If $0<\alpha<2$, then

$$
X_{t}=B_{2 \sigma_{t}}
$$

where $\sigma_{t}$ is a stable subordinator of index $\alpha / 2$ independent of $B_{t}$; see $[6$. Thus

$$
P_{t}^{\alpha}(x, y)=\int_{0}^{\infty} P_{u}^{2}(x, y) g_{\alpha / 2}(t, u) d u
$$

where $g_{\alpha / 2}(t, u)$ is the transition density of $\sigma_{t}$. Thus for every positive $t, P_{t}^{\alpha}(x, y)=$ $f_{t}^{\alpha}(|x-y|)$ and the function $f_{t}^{\alpha}(r)$ is decreasing.

Theorem 3. Suppose $D \subset \mathbf{R}^{2}$ is a convex domain of finite inradius $R_{D}$. Then for any $0<\alpha \leq 2$,

$$
P_{z}\left\{\tau_{D, \alpha}>t\right\} \leq P_{0}\left\{\tau_{S(D), \alpha}>t\right\}=P_{0}\left\{\tau_{I(D), \alpha}>t\right\}
$$

for all $z \in D$ and all $t>0$ where, as above, $I(D)=\left(-R_{D}, R_{D}\right)$ and $S(D)=$ $\mathbf{R} \times I(D)$.

Proof. As in the proof of Theorem 1 either $D$ is contained in a strip of the same inradius, which after rotating and translating if necessary we may assume that it is the standard strip $S(D)$, in which case the inequality is trivial, or it is contained in a triangle of the same inradius. After a translation we may assume that the largest disk contained in the triangle is centered at the origin. Denote this triangle by $T$. Let $T_{k}$ be a sequence of increasing triangles whose closures are strictly contained in $T$ with inradius $R_{D}-\frac{1}{k}$ and whose union is $T$. Denote the corresponding strips 
by $S\left(D_{k}\right)$. By the right continuity of the sample paths and the Markov property of the stable processes, we have, with $z_{0}=z$,

$$
\begin{aligned}
P_{z}\{ & \left.\tau_{T, \alpha}>t\right\}=P_{z}\left\{X_{s} \in T, 0 \leq s \leq t\right\} \\
& =\lim _{k \rightarrow \infty} \lim _{m \rightarrow \infty} P_{z}\left\{X_{\frac{j t}{m}} \in T_{k}, j=1, \ldots, m\right\} \\
& =\lim _{k \rightarrow \infty} \lim _{m \rightarrow \infty} \int_{T_{k}} \ldots \int_{T_{k}} \prod_{j=1}^{m} P_{t / m}^{\alpha}\left(z_{j}-z_{j-1}\right) d z_{1} \cdots d z_{m} \\
& \leq \lim _{k \rightarrow \infty} \lim _{m \rightarrow \infty} \int_{S\left(D_{k}\right)} \ldots \int_{S\left(D_{k}\right)} P_{t / m}^{\alpha}\left(z_{1}\right) \prod_{j=2}^{m} P_{t / m}^{\alpha}\left(z_{j}-z_{j-1}\right) d z_{1} \cdots d z_{m} \\
& =\lim _{k \rightarrow \infty} \lim _{m \rightarrow \infty} P_{0}\left\{X_{\frac{j t}{m}} \in S\left(D_{k}\right), j=1, \ldots, m\right\} \\
& =P_{0}\left\{\tau_{S(D), \alpha}>t\right\},
\end{aligned}
$$

where we have used Theorem 1 and the fact that the transition functions are radially symmetric and decreasing. Note that (11) implies that

$$
P_{0}\left\{\tau_{S(D), \alpha}>t\right\}=P_{0}\left\{\tau_{I(D), \alpha}>t\right\},
$$

and this concludes the proof of Theorem 1.

Let us now denote the Dirichlet heat kernel associated to $X_{t}$ on $D$ by $P_{t}^{D, \alpha}(z, w)$ and its Green's function by $G_{D, \alpha}(z, w)$. The potential theory of these processes has been extensively studied for several years. For some of the recent developments and basic properties of $P_{t}^{D, \alpha}(z, w)$ and $G_{D, \alpha}(z, w)$ we refer the reader to Z-Q. Chen and R. Song [8] and [9]. Inequality (12) is equivalent to

$$
\int_{D} P_{t}^{D, \alpha}(z, w) d w \leq \int_{-R(D)}^{R(D)} P_{t}^{I(D), \alpha}(0, y) d y
$$

for all $z \in D$ and $t>0$. Upon integrating in $t$ this inequality we obtain

$$
\int_{D} G_{D, \alpha}(z, w) d w \leq \int_{-R_{D}}^{R_{D}} G_{I(D), \alpha}(0, w) d w
$$

which is an extension of Sperb's result.

As in the classical case of Brownian motion, if $\lambda_{D, \alpha}$ denotes the first Dirichlet eigenvalue for the operator associated to $X_{t}$ in $D$, it follows from the eigenfunction expansion of the heat kernel $P_{t}^{D, \alpha}(z, w)$ (see [9]) that

$$
-\lambda_{D, \alpha}=\lim _{t \rightarrow \infty} \frac{1}{t} \log P_{z}\left\{\tau_{D, \alpha}>t\right\}
$$

for all bounded domains $D$. Thus (12) implies that

$$
\lambda_{I(D), \alpha} \leq \lambda_{D, \alpha}
$$

for any planar bounded convex domain of finite inradius $R_{D}$. However, as mentioned earlier if the domain is not bounded, then it is contained in a strip of the same inradius and this inequality trivially holds. This gives the extension of Hersh's [12] result to symmetric stable processes. Note that $\lambda_{D, 2}=\lambda_{D}$ with the latter as in the introduction.

When $\alpha=2$ we of course have that

$$
\lambda_{I(D)}=\frac{\pi^{2}}{4 R_{D}^{2}}
$$


and hence Hersh's $[12$ result asserts that

$$
\frac{\pi^{2}}{4 R_{D}^{2}} \leq \lambda_{D}
$$

for all convex domains of inradius $R_{D}$.

Very little seems to be known concerning the explicit value of $\lambda_{I(D), \alpha}$ for $0<$ $\alpha<2$ despite the often appearances of this constant in other problems. (See for example K. Khoshnevisan and Z. Shi [13] for its connections to Chung's law of the iterated logarithm.) Let $B_{r}=B(0, r) \subset \mathbf{R}^{n}$ be the ball centered at the origin of radius $r$. It is proved in [1] that

$$
E_{x}\left(\tau_{B_{r}, \alpha}\right)=K(\alpha, n)\left(r^{2}-|x|^{2}\right)^{\alpha / 2}
$$

where

$$
K(\alpha, n)=\frac{\Gamma\left(\frac{n}{2}\right)}{2^{\alpha} \Gamma\left(1+\frac{\alpha}{2}\right) \Gamma\left(\frac{n+\alpha}{2}\right)} .
$$

If we let $\varphi_{\alpha}(x)$ be the eigenfunction corresponding to the eigenvalue $\lambda_{B_{r}, \alpha}$ it follows that this function is in $L^{\infty}$ and for all $x \in B_{r}$,

$$
\begin{aligned}
\varphi_{\alpha}(x) & =\lambda_{B_{r}, \alpha} \int_{B_{r}} \varphi_{\alpha}(y) G_{B_{r}, \alpha}(x, y) d y \\
& \leq \lambda_{B_{r}, \alpha} \sup _{x \in B_{r}} \varphi_{\alpha}(x) \int_{B_{r}} G_{B_{r}, \alpha}(x, y) d y .
\end{aligned}
$$

Since

$$
E_{x}\left(\tau_{B_{r}}\right)=\int_{B_{r}} G_{B_{r}, \alpha}(x, y) d y \leq K(\alpha, n) r^{\alpha}
$$

we have that

$$
\frac{2^{\alpha} \Gamma\left(1+\frac{\alpha}{2}\right) \Gamma\left(\frac{n+\alpha}{2}\right)}{\Gamma\left(\frac{n}{2}\right) r^{\alpha}} \leq \lambda_{B_{r}, \alpha} .
$$

Taking $n=1$ we obtain the following from (13).

Corollary 1. Let $D \subset \mathbf{R}^{2}$ be a convex domain of inradius $R_{D}$. Then for any $0<\alpha \leq 2$,

$$
\frac{2^{\alpha} \Gamma\left(1+\frac{\alpha}{2}\right) \Gamma\left(\frac{1+\alpha}{2}\right)}{\Gamma\left(\frac{1}{2}\right) R_{D}^{\alpha}} \leq \lambda_{I(D), \alpha} \leq \lambda_{D, \alpha} \leq \lambda_{B_{R_{D}}, \alpha} .
$$

When $\alpha=2$ and $R_{D}=1$ we get the inequality $2 \leq \lambda_{D}$ which of course is not as good as the sharp estimate $\pi^{2} / 4 \leq \lambda_{D}$. For an upper bound we have the following general theorem.

Theorem 4. Let $D \subset \mathbf{R}^{n}$ be a domain of finite volume. Then $\lambda_{D, \alpha} \leq\left(\lambda_{D}\right)^{\frac{\alpha}{2}}$.

This result together with (14) and the fact that the first eigenvalue for the unit disk is the square of the smallest positive zero of the Bessel function of order 0, denoted by $j_{0}$, gives

Corollary 2. Let $D \subset \mathbf{R}^{2}$ be a convex domain of inradius $R_{D}$. Then for any $0<\alpha \leq 2$,

$$
\frac{2^{\alpha} \Gamma\left(1+\frac{\alpha}{2}\right) \Gamma\left(\frac{1+\alpha}{2}\right)}{\Gamma\left(\frac{1}{2}\right) R_{D}^{\alpha}} \leq \lambda_{D, \alpha} \leq \frac{j_{0}^{\alpha}}{R_{D}^{\alpha}}
$$


Proof of Theorem 4. Let $x \in D$. Since $X_{t}$ has right-continuous paths and $\sigma_{t}$ is nondecreasing we have

$$
\begin{aligned}
P_{x}\left\{\tau_{D, \alpha}>t\right\} & =P_{x}\left\{X_{s} \in D, 0 \leq s \leq t\right\} \\
& =P_{x}\left\{B_{2 \sigma_{s}} \in D, 0 \leq s \leq t\right\} \\
& \geq P_{x}\left\{\tau_{D, 2}>\sigma_{s}, 0 \leq s \leq t\right\} \\
& =P_{x}\left\{\tau_{D, 2}>\sigma_{t}\right\} .
\end{aligned}
$$

Since $\sigma_{t}$ and $B_{t}$ are independent,

$$
P_{x}\left\{\tau_{D, \alpha}>t\right\} \geq P_{x}\left\{\tau_{D, 2}>\sigma_{t}\right\}=\int_{0}^{\infty} P_{x}\left\{\tau_{D, 2}>a\right\} g_{\alpha / 2}(t, a) d a .
$$

Let $\left\{\varphi_{k}\right\}_{1}^{\infty}$ be an orthonormal set of eigenfunctions for $-\Delta$ in $D$ with eigenvalues $\left\{\lambda_{k}\right\}_{1}^{\infty}$, so that with the above notation $\lambda_{1}=\lambda_{D}$. Then

$$
P_{x}\left\{\tau_{D, 2}>t\right\}=\sum_{k=1}^{\infty} e^{-\lambda_{k} t} \varphi_{k}(x) \int_{D} \varphi_{k}(y) d y,
$$

where the series converges absolutely and uniformly in $[\delta, \infty) \times D$, for every $\delta>0$. Indeed let $\delta>0$; since $P_{s}^{D}(x, y) \leq(4 \pi s)^{-n / 2}$ for all $s>0$ we see that

$$
\begin{aligned}
\left|e^{-\lambda_{k} s} \varphi_{k}(x)\right| & =\left|\int_{D} P_{s}^{D}(x, y) \varphi_{k}(y) d y\right| \\
& \leq(4 \pi s)^{-n / 2}\left\|\varphi_{k}\right\|_{2}(\operatorname{vol}(D))^{1 / 2} \\
& =(4 \pi s)^{-n / 2}[\operatorname{vol}(D)]^{1 / 2} .
\end{aligned}
$$

Taking $s=1 / \lambda_{k}$ in this inequality gives that

$$
\left|\varphi_{k}(x)\right| \leq e(4 \pi)^{-n / 2} \lambda_{k}^{n / 2}[\operatorname{vol}(D)]^{1 / 2}
$$

for all $x \in D$. Thus the fact that the volume of the domain is finite implies that there exists $C>0$ such that

$$
\sum_{k=1}^{\infty} e^{-\lambda_{k} t} \varphi_{k}(x) \int_{D} \varphi_{k}(y) d y \leq C \sum_{k=1}^{\infty} e^{-\lambda_{k} \delta / 2}
$$

for all $(t, x) \in[\delta, \infty) \times D$. This quantity, however, is nothing more than the trace of the semigroup associated with the Dirichlet Laplacian in $D$ which is finite, since the volume of $D$ is finite (see [10]), and the Weierstrass M-test is applicable.

Since the Laplace transform of $\sigma_{t}$ is $e^{-t u^{\alpha / 2}}$ (see [11]), we obtain from (15) that

$$
P_{x}\left\{\tau_{D, \alpha}>t\right\} \geq \sum_{k=1}^{\infty} e^{-\lambda_{k}^{\alpha / 2} t} \varphi_{k}(x) \int_{D} \varphi_{k}(y) d y,
$$

and again we claim that the series is absolutely and uniformly convergent on $[\delta, \infty) \times$ $D$. As above, it is enough to prove the convergence of the series $\sum_{k=1}^{\infty} e^{-\lambda_{k}^{\alpha / 2} \delta / 2}$. This time, however, we need to be a little more careful. By Weyl's asymptotic formula (see [7]) we have $c_{n, D} k^{n / 2} \leq \lambda_{k}$ where $c_{n, D}$ is a constant that depends on $n$ and on the volume of the domain $D$. This proves the desired convergence. From (17) we conclude that

$$
\lambda_{D}^{\alpha / 2}=\lambda_{1}^{\alpha / 2} \geq-\lim _{t \rightarrow \infty} \frac{1}{t} \log P_{x}\left\{\tau_{D, \alpha}>t\right\}=\lambda_{D, \alpha},
$$

which completes the proof. 
If instead of fixing the inradius we fix the volume, then the Brascamp-LiebLuttinger Theorem 2 and the above arguments give

Theorem 5. Let $D \subset \mathbf{R}^{n}, n \geq 1$, be a domain of finite volume. Let $D^{*}$ be the ball centered at the origin with $\operatorname{vol}(D)=\operatorname{vol}\left(D^{*}\right)$. Then for all $0<\alpha \leq 2, z \in D$ and $t>0$

$$
P_{z}\left\{\tau_{D, \alpha}>t\right\} \leq P_{0}\left\{\tau_{D^{*}, \alpha}>t\right\}
$$

and

$$
\frac{\gamma_{n}^{\alpha / n} 2^{\alpha} \Gamma\left(1+\frac{\alpha}{2}\right) \Gamma\left(\frac{n+\alpha}{2}\right)}{\Gamma\left(\frac{n}{2}\right) \operatorname{vol}(D)^{\alpha / n}} \leq \lambda_{D^{*}, \alpha} \leq \lambda_{D, \alpha},
$$

where $\gamma_{n}$ is the volume of the unit ball in $\mathbf{R}^{n}$.

This time, however, one does not have an upper bound even in the case of the Laplacian.

\section{ACKNOWLEDGEMENT}

We would like to thank the referee for many useful comments and corrections.

\section{REFERENCES}

[1] C. Bandle, Isoperimetric Inequalities and Applications, Monographs and Studies in Mathematics, 7, Pitman 1980. MR 81e:35095

[2] R. Bañuelos and T. Carroll, Brownian motion and the fundamental frequency of a drum, Duke Math. J. 75 (1994), 575-602. MR 96m:31003

[3] R. Bañuelos and E. Housworth, An isoperimetric-type inequality for integrals of Green's functions, Michigan Math. J. 42 (1995), 603-611. MR 96j:30038

[4] R. Bañuelos and P. Kröger, Isoperimetric-type inequalities for solutions of the heat equation, Indiana Math. J. 46 (1997), 83-91. MR 98k:35081

[5] H.J. Brascamp, E. H. Lieb and J.M. Luttinger, A General Rearrangement Inequality for Multiple Integrals, Jour. Funct. Anal. 17 (1974), 227-237. MR 49:10835

[6] R.M. Blumenthal and R.K. Getoor, Some Theorems on Stable Processes, Trans. Amer. Soc. 95 (1960), 263-273. MR 22:10013

[7] I. Chavel, Eigenvalues in Riemannian geometry, Academic Press, (1984). MR 86g:58140

[8] Z-Q. Chen and R. Song, Estimates on Green functions and Poisson kernels of symmetric stable processes in bounded domains. Math. Ann. 312 (1998), 465-501. MR 2000b:60179

[9] Z-Q. Chen and R. Song, Intrinsic ultracontractivity and conditional gauge for symmetric stable processes, J. Funct. Anal. 150 (1997), 204-239. MR 98j:60103

[10] E.B. Davies, Heat kernels and Spectral Theory, Cambridge University Press, Cambridge, (1989). MR 90e:35123

[11] R. K. Getoor, First passage time for symmetric stable processes in space, Trans. Amer. Math. Soc. 101 (1961), 75-90. MR 25:604

[12] J. Hersh, Sur la fréquence fondamentale d'une membrane vibrante: évaluations par défaut et principe de maximum, Z. Angew. Mech. 11 (1960), 387-441.

[13] D. Khoshnevisan and Z. Shi, Chung's Law for Integrated Brownian Motion, Trans. Amer. Math. Soc. 350 (1998), 4253-4264. MR 98m:60056

[14] P. Kröger, On the spectral gap for compact manifolds J. Differ. Geom. 36 (1992), 315-330. MR 94g:58236

[15] J. M. Luttinger, Generalized isoperimetric inequalities, J. Math. Phys. 14 (1973), 586-593. MR 49:1969

[16] _ Generalized isoperimetric inequalities. II J. Math. Phys. 14 (1973), 1444-1447. MR 49:6012

[17] Generalized isoperimetric inequalities. III J. Math. Phys. 14 (1973), 1448-1450. MR 49:6012 
[18] M. H. Protter, Lower bound for the fundamental frequency of a convex region Proc. Amer. Math. Soc. 81 (1981), 65-70. MR 82b:35113

[19] R. Sperb, Maximum Principles and Their Applications, Academic Press, New York 1981. MR 84a:35033

Department of Mathematics, Purdue University, West Lafayette, Indiana 47907

E-mail address: banuelos@math.purdue.edu

Institute of Mathematics, Warsaw University, Banacha 2, 02-097 Warszawa, Poland

E-mail address: rlatala@mimuw.edu.pl

Department of Mathematics, Purdue University, West Lafayette, Indiana 47907

E-mail address: mendez@math.purdue.edu 